Pacific Journal of Mathematics

THE DUAL OF A SPACE WITH THE RADON-NIKODÝM
PROPERTY

James Bryan Collier 


\title{
THE DUAL OF A SPACE WITH THE RADON-NIKODYM PROPERTY
}

\author{
JAMES B. Collier
}

\begin{abstract}
Two characterizations of a Banach space with the Radon-Nikodym property are proved here. The first shows its equivalence with a condition on the dual space which is somewhat weaker than that of being an Asplund space. This leads to a second characterization by a renorming property.
\end{abstract}

A convex function $f$ on a Banach space $X$ will be assumed to take its values in $(-\infty,+\infty]$ and to be finite at some point. The domain of continuity of $f$ is the convex open set of all points at which $f$ is finite and continuous. The space $X$ is called an Asplund space if each convex function on $X$ is Fréchet differentiable on a dense $G_{\delta}$ subset of its domain of continuity. If $X$ is the dual of a Banach space $Y$, then it will be called a weak*-Asplund space if each weak* lower semi-continuous $\left(w^{*}\right.$-lsc) convex function on $X$ is Fréchet differentiable on a dense $G_{\delta}$ subset of its domain of continuity. The terms " $G_{\delta}$ " and "domain of continuity" here still refer to the norm topology on $X$. Thus a dual space which is an Asplund space is also a weak*-Asplund space. A Banach space may be said to have the Radon-Nikodym property (RNP) if each closed bounded convex subset is the closed convex hull of its strongly exposed points [6]. A point $x$ in a set $C$ is said to be strongly exposed by a linear functional $y$ if the supremum of $y$ over $C$ is finite and attained at $x$ and $\left\|x_{i}-x\right\| \rightarrow 0$ whenever $\left\{x_{i}\right\}$ is a sequence in $C$ for which $y\left(x_{\imath}\right) \rightarrow y(x)$.

Using the same method as in [3], we characterize the dual of a space with the RNP by its differentiability properties. This allows us to give an alternate proof of a result of Huff and Morris [4] concerning the density of strongly exposing functionals and to observe that weak*Asplund spaces enjoy some of the permanence properties that Asplund spaces do.

THEOREM 1. A Banach space $X$ has the RNP if and only if $X^{*}$ is a weak*-Asplund space.

Proof. Assume $X$ has the RNP and let $f$ be a $w^{*}$-lsc convex function on $X^{*}$ with nonempty domain of continuity $D$. Choose any point $w \in D$ and an $\varepsilon>0$ so that $f$ is bounded on $N=\{y:\|y-w\| \leqq \epsilon\}$ and $N \subseteq D$. We use the dual norm so that $N$ is weak* closed. Define $g$ 
on $X^{*}$ by $g(y)=f(y)$ if $y \in N$ and $g(y)=+\infty$ otherwise. Then $g$ is a $w^{*}$-lsc convex function on $X^{*}$, bounded on $N$, and whose domain of continuity is the interior of $N$. We may assume without loss of generality that the unit ball $B$ of $X^{*}$ is contained in $N$ and $-1 \leqq g(y) \leqq 0$ for all $y \in N$. Choose some $\lambda>1$ such that $N \subseteq \lambda B$.

Define $p$ on $X^{*}$ by $p(y)=0$ if $y \in B$ and $p(y)=+\infty$ otherwise. Let $q(y)=p(y / \lambda)-1$. Then $p$ and $q$ are $w^{*}$-lsc convex functions on $X^{*}$. For any convex function $h$ on $X^{*}$, the conjugate of $h$ on $X$ is $h^{*}(x)=\sup \left\{y(x)-h(y): y \in X^{*}\right\}$ for each $x \in X$. Thus $p^{*}(x)=\|x\|$ and $q^{*}(x)=\lambda\|x\|+1$. Since $q(y) \leqq g(y) \leqq p(y)$ for all $y \in X^{*}, p^{*}(x) \leqq$ $g^{*}(x) \leqq q^{*}(x)$ for all $x \in X$, and hence $\|x\| \leqq g^{*}(x) \leqq \lambda\|x\|+1$ for all $x \in X$. This implies that the closed convex set $C=\left\{x \in X: g^{*}(x) \leqq 2\right\}$ is bounded and has nonempty interior.

Let epi $g^{*}=\left\{(x, r): x \in X, g^{*}(x) \leqq r\right\}, H=\{(x, r): x \in X, r \geqq 2\}$ and $K=$ epi $g^{*} \cap H$. Since $K \subseteq C \times[0,2], K$ is a closed bounded convex subset of $X \times \mathbf{R}$ with nonempty interior. It is well-known that the RNP is preserved under products; hence $X \times \mathbf{R}$ has the RNP and $K$ must be the closed convex hull of its strongly exposed points. As a consequence, there must be a point $a$ in the interior of $C$ such that $g^{*}(a)<2$ and $(a, g(a))$ is strongly exposed as a point of $K$ by some functional $(b,-1) \in X^{*} \times \mathbf{R}$. Since $g^{*}(a)<2$ and epi $g$ is convex, $(a, g(a))$ is also strongly exposed as a point of epi $g$ by $(b,-1)$. Because $g$ is $w^{*}$-lsc, Theorem 1 in [2, p. 450] together with the Lemma in [3] implies that $g$ is Fréchet differentiable at $b$ with gradient $a$. Since $b$ lies in the interior of $N, f$ is also Fréchet differentiable at $b$ and $\|w-b\|<\epsilon$. Since the choice of $w \in D$ and $\epsilon>0$ was arbitrary, the set $G$ of points at which $f$ is Fréchet differentiable is dense in $D$. Lemma 6 in [1, p. 43] implies that $G$ must in fact be a dense $G_{\delta}$ subset of $D$ and therefore $X^{*}$ is a weak*-Asplund space.

Assume now that $X^{*}$ is a weak*-Asplund space and $C$ is a closed bounded convex set in $X$. Define $f(x)=0$ if $x \in C$ and $f(x)=+\infty$ otherwise. Then $f^{*}(y)=\sup \{y(x)-f(x): x \in X\}$ is a $w^{*}$-lsc convex function on $X^{*}$ whose domain of continuity is $X^{*}$. Since $X^{*}$ is a weak*-Asplund space, $f^{*}$ is Fréchet differentiable on a dense $G_{\delta}$ subset $G$ of $X^{*}$. From Theorem 1 in [2, p. 450] it follows that each functional in $G$ strongly exposes a point of $C$. The density of $G$ implies that $C$ is the closed convex hull of its strongly exposed points and hence $X$ has the RNP.

The last part of the proof of Theorem 1 actually proves the following result of Huff and Morris [4]:

COROLlARY 2. If $X$ has the RNP and $C$ is a closed bounded convex subset, then the set of linear functionals which strongly expose some point of $C$ is a dense $G_{\delta}$ subset of $X^{*}$. 
The convexity restriction on $C$ which occurs in this proof is easily dropped by observing that a linear functional strongly exposes a point of a closed bounded set $A$ whenever it strongly exposes a point of the closed convex hull of $A$.

Since the dual norm on a dual space is a $w^{*}$-lsc convex function, the following is also an immediate consequence of Theorem 1:

Corollary 3. If $X$ has the RNP, then the dual norm on $X^{*}$ is Fréchet differentiable on a dense $G_{\delta}$ subset of $X^{*}$.

The differentiability of the dual norm can be used to characterize spaces with the RNP. Let $A$ be a nonempty bounded subset of a Banach space $X$. A slice of $A$ will be any set of the form $S(A, y, \epsilon)=$ $\{x \in A: y(x)+\epsilon>\sup y[A]\}$ where $y \in X^{*}$ and $\epsilon>0$. We can show the following:

THEOREM 4. A Banach space $X$ fails to have the RNP if and only if there is an equivalent norm on $X$ for which the dual norm on $X^{*}$ is Fréchet differentiable nowhere.

Proof. If such a renorming exists, then Corollary 3 implies that $X$ cannot have the RNP. In order to prove the other direction assume that for each equivalent norm on $X$, the dual norm on $X^{*}$ is Fréchet differentiable at some point. Let $C$ be any closed bounded convex subset of $X$ and let $B$ be the unit ball. Let $D$ be the closure of $C+B$ and let $E$ be the closure of $D+(-D)$, then $E$ is the unit ball of an equivalent norm on $X$.

Define $f$ on $X$ by $f(x)=0$ is $x \in E$ and $f(x)=+\infty$ otherwise, then the conjugate of $f, f^{*}(y)=\sup \{y(x)-f(x): x \in X\}$, is the corresponding dual norm on $X^{*}$. By hypothesis, $f^{*}$ is Fréchet differentiable at some point $b$ with gradient $a \in X^{* *}$. Since $f^{*}$ is $w^{*}$-lsc, Corollary 5 in [2] implies that $a$ actually belongs to $X$. By Theorem 1 in [2] $f$ is norm rotund at $a$ relative to $b$ and therefore $E$ is strongly exposed at $a$ by $b$. Thus diam $S(E, b, \epsilon) \rightarrow 0$ as $\epsilon \rightarrow 0$. By the construction of $E$ it follows that $\operatorname{diam} S(E, b, \epsilon) \geqq \operatorname{diam} S(C, b, \epsilon)$ and hence $\operatorname{diam} S(C, b, \epsilon) \rightarrow 0$ as $\epsilon \rightarrow 0$ also. Since each closed bounded convex set $C$ is dentable in the sense defined by Rieffel [7], $X$ must have the RNP and the theorem follows.

A number of permanence properties for weak*-Asplund spaces may be proved using Theorem 1 in very much the same fashion as the permanence properties for Asplund spaces were proved in [5].

THEOREM 5. If $X^{*}$ and $Y^{*}$ are weak ${ }^{*}$-Asplund spaces, then $X^{*} \times$ $Y^{*}$ is weak ${ }^{*}$-Asplund. 
Proof. Theorem 1 implies that both $X$ and $Y$ have the RNP and hence $X \times Y$ has the RNP. Therefore $(X \times Y)^{*}$, which is isomorphic to $X^{*} \times Y^{*}$, is weak*-Asplund.

THEOREM 6. If $X^{*}$ is a weak ${ }^{*}$-Asplund space and $M$ is a weak* closed subspace of $X^{*}$, then $X^{*} / M$ is weak ${ }^{*}$-Asplund.

Proof. Let $M^{\perp}=\{x \in X: y(x)=0$ for all $y \in M\}$ be a closed subspace of $X$. Theorem 1 implies that $X$ has the RNP and hence $M^{\perp}$ has the RNP also. Therefore $\left(M^{\perp}\right)^{*}$, which is isomorphic to $X^{*} / M$, is weak*-Asplund.

Namioka and Phelps [5] raised the question of whether a Banach space $X$ is an Asplund space whenever $X^{*}$ has the RNP. This may now be restated in the following way: If $X^{* *}$ is a weak*-Asplund space, is $X$ an Asplund space? The converse is known to be true. If we consider $X$ to be a (norm) closed subspace of $X^{* *}$ by the usual embedding, then each continuous convex function defined on an open convex subset of $X$ is the restriction of a $w^{*}$-lsc convex function on $X^{* *}$. We note, however, that since the RNP is not preserved under quotients, we cannot expect even a weak* closed subspace of a weak*-Asplund space to thave good differentiability properties.

\section{REFERENCES}

1. E. Asplund, Fréchet differentiability of convex functions, Acta Math., 121 (1968), 31-47.

2. E. Asplund and R. T. Rockafellar, Gradients of convex functions, Trans. Amer. Math. Soc., 139 (1969), 433-467.

3. J. B. Collier, A class of strong differentiability spaces, to appear.

4. R. E. Huff and P. D. Morris, Geometric characterizations of the Radon-Nikodym property in Banach spaces, to appear.

5. I. Namioka and R. R. Phelps, Banach spaces which are Asplund spaces, to appear.

6. R. R. Phelps, Dentability and extreme points in Banach spaces, J. Functional Anal., 17 (1974), 78-90.

7. M. A. Rieffel, Dentable subsets of Banach spaces with applications to a Radon-Nikodym theorem, Proc. Conf. Functional Analysis, Thompson Book Co., Washington, D.C. (1967), 71-77.

Received September 18, 1975.

University of Southern California, Los Angeles 



\section{Pacific Journal of Mathematics}

Vol. 64, No. 1

May, 1976

Walter Allegretto, Nonoscillation theory of elliptic equations of order $2 n \ldots \ldots \quad 1$

Bruce Allem Anderson, Sequencings and starters.................. 17

Friedrich-Wilhelm Bauer, A shape theory with singular homology .......... 25

John Kelly Beem, Characterizing Finsler spaces which are

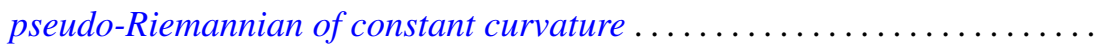

Dennis K. Burke and Ernest A. Michael, On certain point-countable

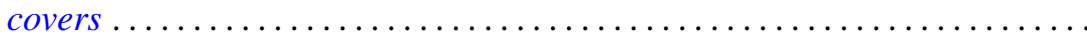

Robert Chen, A generalization of a theorem of Chacon ............... 93

Francis H. Clarke, On the inverse function theorem ................ 97

James Bryan Collier, The dual of a space with the Radon-Nikodým

property ....................................... 103

John E. Cruthirds, Infinite Galois theory for commutative rings ............ 107

Artatrana Dash, Joint essential spectra......................... 119

Robert M. DeVos, Subsequences and rearrangements of sequences in FK

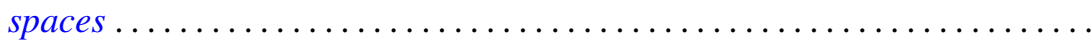

Geoffrey Fox and Pedro Morales, Non-Hausdorff multifunction generalization

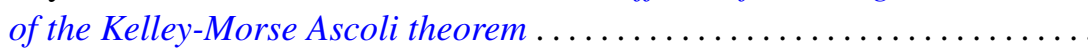

Richard Joseph Fleming, Jerome A. Goldstein and James E. Jamison, One

parameter groups of isometries on certain Banach spaces.............

Robert David Gulliver, II, Finiteness of the ramified set for branched

immersions of surfaces

Kenneth Hardy and István Juhász, Normality and the weak cb property ...... 167

C. A. Hayes, Derivation of the integrals of $L^{(q)}$-functions.

Frederic Timothy Howard, Roots of the Euler polynomials .

Robert Edward Jamison, II, Richard O'Brien and Peter Drummond Taylor, On

embedding a compact convex set into a locally convex topological vector space ....................................

Andrew Lelek, An example of a simple triod with surjective span smaller than span ...

Janet E. Mills, Certain congruences on orthodox semigroups

Donald J. Newman and A. R. Reddy, Rational approximation of $e^{-x}$ on the positive real axis.

John Robert Quine, Jr., Homotopies and intersection sequences ...

Nambury Sitarama Raju, Periodic Jacobi-Perron algorithms and fundamental units ....

Herbert Silverman, Convexity theorems for subclasses of univalent functions. . .

Charles Frederick Wells, Centralizers of transitive semigroup actions and endomorphisms of trees.........................

Volker Wrobel, Spectral approximation theorems in locally convex spaces ..................... 